\title{
A concerted mechanism for berberine bridge enzyme
}

\author{
Andreas Winkler ${ }^{1}$, Andrzej Łyskowski ${ }^{2,}$, Sabrina Ried1 ${ }^{1, \#}$, Martin Puhl ${ }^{1}$, \\ Toni M. Kutchan ${ }^{3}$, Peter Macheroux ${ }^{* 1} \&$ Karl Gruber ${ }^{* 2}$
}

${ }^{1}$ Institute of Biochemistry, Graz University of Technology, Petersgasse 12/II, 8010 Graz, Austria

${ }^{2}$ Institute of Molecular Biosciences, University of Graz, Humboldtstraße 50/III, 8010 Graz, Austria

${ }^{3}$ Donald Danforth Plant Science Center, 975 North Warson Road, St. Louis, Missouri 63132, USA

*peter.macheroux@tugraz.at, phone: (+43 316) 873-6450, fax: (+43 316) 873-6952;

karl.gruber@uni-graz.at, phone: (+43 316) 380-5483, fax: (+43 316) 380-9897

present addresses: ${ }^{\star}$ Institute of Biotechnology, University of Helsinki, Finland; ${ }^{\#}$ Institute of Biophysics and X-ray Structure Research, Austrian Academy of Sciences, Austria 
Berberine bridge enzyme (BBE) catalyzes the conversion of $(S)$-reticuline to $(S)$ scoulerine by formation of a carbon-carbon bond between the $\mathrm{N}$-methyl group and the phenolic ring. We elucidated the structure of BBE from Eschscholzia californica and determined the kinetic rates for three active site protein variants. Here, we propose a catalytic mechanism combining base-catalyzed proton abstraction with concerted $\mathrm{C}-\mathrm{C}$ coupling accompanied by hydride transfer from the $\mathrm{N}$-methyl group to the N5 atom of the FAD cofactor.

Berberine bridge enzyme (BBE) plays a central role in benzophenanthridine biosynthesis and channels its substrate (S)-reticuline (1) towards berberine (2) and sanguinarine (3) generation ${ }^{1}$. BBE catalyzes the stereospecific conversion of $(S)$-reticuline to $(S)$-scoulerine (4) forming the so called berberine bridge $(\mathrm{C} 8)$ by linking the isoquinoline ring with the benzyl ring system (Fig. 1a) ${ }^{1}$. This cyclization reaction has no known equivalent in organic chemistry and is proposed to proceed via a two step process starting with oxidation of the Nmethyl group to the corresponding iminium ion followed by an ionic ring closure initiated by the deprotonated C3'-OH group of the benzyl moiety ${ }^{2}$. This hydroxyl group apparently plays an important role for the enzymatic reaction, as substrate analogs lacking this group are not processed by the enzyme ${ }^{2}$. This finding suggests that an active site base may be involved in the reaction mechanism by deprotonation of the hydroxyl group thereby facilitating the nucleophilic attack of the $\mathrm{C} 2$ ' carbon onto the $\mathrm{N}$-methyl group. A similar reaction mechanism involving the intermediate formation of an iminium species is favored for amine oxidases, a diverse group of flavin-dependent enzymes (reviewed in refs. 3-5).

In order to better understand the mechanism of action for the BBE catalyzed reaction, we elucidated the three-dimensional x-ray crystal structure of the enzyme from Eschscholzia californica in two different crystal forms (monoclinic and tetragonal) as well as in complex with the natural substrate (S)-reticuline (see Supplementary Methods, Supplementary Discussion and Supplementary Table 1 online). Based on this structure, we initiated a mutagenesis program involving three active-site amino acids and analyzed their function.

The resulting molecular structure comprises two domains - an FAD (5) binding domain and an $\alpha / \beta$-domain with a seven stranded, anti-parallel $\beta$-sheet forming the substrate binding site (Fig. 1b) - showing BBE to belong to the $p$-cresol methylhydroxylase (PCMH) superfamily (Supplementary Fig 1 online). The structure also confirms the previously identified ${ }^{6}$ bicovalent attachment of the flavin cofactor to N1 of His104 (via C8 $\alpha$ ) and to Cys166 (via C6) 
(Fig. 1c and Supplementary Fig. 2 online), thus providing an additional example for the recently discovered group of bi-covalently flavinylated enzymes ${ }^{6-10}$. The active site cavity close to the cofactor is lined by mainly hydrophobic residues with the only notable exceptions being Tyr106, Thr358, Asn390, Glu417 and His459 (Fig. 1c). The latter two residues show considerable conformational flexibility: Glu-417 is present in two alternate conformations in the monoclinic structure (Fig 1c), whereas His-459 shows two conformations in the tetragonal structure (Supplementary Fig. 2 online). During the course of crystallization, we observed a bleaching of the yellow colored crystals. In line with that, we found flavin electron density indicating the (partial) presence of a colorless $4 a$-spirohydantoin degradation $\operatorname{product}^{11,12}(\mathbf{6})$ most notably in the much slower growing monoclinic crystals (see Supplementary

Discussion and Supplementary Fig. 2 online).

We obtained the structure of the complex with $(S)$-reticuline by soaking a monoclinic crystal in a cryo-solution containing $20 \mathrm{mM}$ of the substrate. Despite the lower resolution of the diffraction data (2.8 compared to $1.65 \AA$ for the unbound structure), we observed clear difference electron density in the binding site (Supplementary Fig. 3 online) thus allowing the unequivocal positioning of the substrate (Fig. 1d). (S)-Reticuline is bound in a deep cleft with the phenolic ring pointing towards the bottom of the binding site (Supplementary Fig. 3 online) and is sandwiched between the flavin cofactor and amino acid residues extending from the $\beta$-sheet of the central domain (Fig. 1b). The active site residue Glu417 is hydrogen bonded to the $\mathrm{C} 3$ '-OH group of the substrate and the carboxamide group of Asn390 interacts with both the $\mathrm{OH}$ - and the 4'-methoxy-group of the phenolic ring (Fig. 1d). The corresponding polar substituents of the isoquinoline moiety are positioned close to Asp352 at the entrance to the active site. In the conformation observed in the complex structure, the Nmethyl group of $(S)$-reticuline is sequestered between the $\mathrm{C} 2$ '-atom of the substrate and the N5-atom of the cofactor with distances of $3.3 \AA$ and $3.1 \AA$ respectively (Fig. 1d). The angle between these three atoms is approximately $130^{\circ}$.

Based on the structural information, we selected three amino acids (Tyr106, Glu417 and His459) for site directed mutagenesis. In contrast to Glu417, Tyr106 and His459 are not directly interacting with the substrate. The estimated $\mathrm{pKa}$ of Tyr106, however, is in the range of the $\mathrm{pH}$ optimum of $\mathrm{BBE}^{1}$ and the proximity of His459 to the other selected residues (Fig. 1d) may modulate their properties. All three muteins could be expressed in roughly similar amounts as the wild-type protein ${ }^{6}$. Spectral characterization of the muteins indicated that the 
bi-covalent flavin linkage was not affected by any of the three amino acid replacements ${ }^{6}$ and that the proteins were fully loaded with cofactor (see Supplementary Discussion).

We analyzed the catalytic efficiency of the protein variants by measuring steady-state turnover rates as well as reductive and oxidative rates in comparison to the wild-type enzyme (Table 1). The most significant change in the observed rates was found for the E417Q mutein (see Supplementary Fig. 4 online), whereas Y106F and H459A showed only minor alterations (Supplementary Discussion). In E417Q the reductive rate was reduced 1500-fold compared to the wild-type enzyme. Cofactor reduction became the rate-limiting step during turnover, but no effect on reoxidation of the cofactor was observed (Table 1). This finding clearly supports the role of the glutamate side chain as the catalytic base necessary for C-C bond formation - consistent with the structure of the substrate complex (Fig. 1d) - and also demonstrates its involvement in the oxidative part of the reaction. The residual activity of this variant is most likely due to a fraction of deprotonated substrate molecules (estimated $\mathrm{pK}_{\mathrm{a}}$ of C3'-OH: 9.5) present at the $\mathrm{pH}$ of the measurement (Table 1).

The essential role of proton abstraction for substrate oxidation was further corroborated by the approx. 30,000-times slower reduction of the cofactor by the substrate analog laudanosine (7) bearing a methoxy instead of a hydroxyl group at the C3'-position (Supplementary Fig. 5 online). The appearance of an absorbance maximum around $360 \mathrm{~nm}$ indicated the formation of a double bond in conjugation to the aromatic ring system of the isoquinoline moiety suggesting that the $\mathrm{C} 1-\mathrm{N}$ bond rather than the exocyclic $\mathrm{N}-\mathrm{CH}_{3}$ bond is oxidized. This type of reactivity was verified by NMR studies for the substrate analog 6,7-dimethoxy-2-methyl1,2,3,4-tetrahydroisoquinoline (8), the conversion of which is accompanied by similar spectral changes (Supplementary Discussion).

Small amounts of formaldehyde (9, approx. 2\% during prolonged incubation times) arise in the conversion of the substrate analog $(S)-\mathrm{N}$-methylcoclaurine $(\mathbf{1 0})^{2}$, which also lacks the C3'$\mathrm{OH}$ group. This finding is considered supporting evidence for the two-step mechanism ${ }^{2}$. In light of our results with laudanosine and $\mathbf{8}$, however, it could also be explained by the formation of the intracyclic imine, followed by tautomerisation and hydrolytic demethylation to yield formaldehyde and $(S)$-coclaurine (11).

The fact that Glu417 is an essential amino acid for substrate oxidation has further interesting mechanistic implications. Since this amino acid does not interact with the N-methyl group but rather with the more distant $\mathrm{C} 3$ '-OH group of the substrate, it is reasonable to propose that deprotonation of the phenolic $\mathrm{OH}$ group increases the nucleophilicity of the $\mathrm{C} 2$ '-atom, thereby 
facilitating an $\mathrm{S}_{\mathrm{N}} 2$-type attack onto the $\mathrm{N}$-methyl group. This leads to $\mathrm{C}$-C bond formation and concomitant transfer of a hydride to the flavin. Hence, flavin reduction and berberine bridge formation are likely to be concerted processes initiated by proton abstraction of the phenolic hydroxyl group (Scheme 1). Even though the N-methyl group of the substrate is bound in proximity of the N5 atom of FAD (Fig. 1d) and is appropriately positioned for hydride transfer (similar as in other flavin-dependent amine oxidases ${ }^{13,14}$ ), the E417Q mutant protein does not significantly catalyze flavin reduction (Table 1), which provides clear evidence for this concerted mechanism. Similarly, the inability of wild-type BBE to efficiently oxidize the substrate analog laudanosine also underlines the importance of the interaction of Glu417 with the C3'-OH group of (S)-reticuline. Unless significant protein dynamics are involved, the observed mode of substrate binding also excludes the proposed mechanism for amine oxidation observed in monoamine and polyamine oxidases ${ }^{3,15}$ including the formation of a covalent adduct between flavin and substrate, since the amine function is too distant from the $\mathrm{C} 4 \mathrm{a}$ atom (approx. $5 \AA$ ) of the cofactor for this type of mechanism (see Fig. 1d). Therefore, the BBE catalyzed reaction cannot involve an iminium intermediate as in the two-step mechanism proposed in previous studies ${ }^{2}$ and hence BBE mechanistically diverges from other amine oxidases ${ }^{3-5}$.

We also found that replacement of Glu417 by glutamine leads to a loss of regiospecificity. Wild-type BBE and all other mutant proteins analyzed so far converted (S)-reticuline only to $(S)$-scoulerine ${ }^{2}$ and produced no potential side-product, e.g. via C-C coupling involving C6' instead of C2' after a rotation of the phenyl ring. Substrate conversion by the E417Q mutein, however, yielded an additional compound amounting to about one third of the total product. This side-product coeluted with an authentic standard of $(S)$-coreximine (12), the product analog obtained after a $180^{\circ}$ rotation of the phenolic ring (Supplementary Fig. 6 online). Through a combined structural and biochemical approach, we are now in a position to propose a new reaction mechanism (Scheme 1) that entails proton abstraction of the $\mathrm{C} 3$ '-OH group by the carboxyl group of Glu417 leading to $\mathrm{S}_{\mathrm{N}} 2$-type attack onto the $\mathrm{N}$-methyl group resulting in the formation of the berberine bridge (Fig. 1a). C-C bond formation is associated with expulsion of a hydride from the methyl group to the flavin N5 position leading to a twoelectron reduction of the cofactor. In contrast to previous findings ${ }^{2}$ this mechanistic proposal incorporates both oxidation and ring closure into a single concerted step. The $\mathrm{S}_{\mathrm{N}} 2$-type mechanism is in agreement with earlier studies demonstrating inversion of configuration at the N-methyl group ${ }^{16}$. The proposed hydride transfer to the flavin is in line with the absence 
of any flavin radical intermediate during substrate mediated reduction of the enzyme in stopped-flow experiments ${ }^{17}$ (see also Supplementary Fig. 4 online).

These new mechanistic ideas are potentially also relevant for two closely related enzymes: $\Delta^{1}$ tetrahydrocannabinolic acid (THCA) synthase and cannabidiolic acid (CBDA) synthase from Cannabis sativa ${ }^{18,19}$. Both enzymes also catalyze an oxidative $\mathrm{C}-\mathrm{C}$ coupling reaction, which is proposed to proceed via a two-step mechanism, where ring closure is an ionic process following the oxidation of the substrate ${ }^{18,19}$. Due to the similarity of the catalyzed reactions and the significant sequence similarity to BBE ( $40 \%$ identity), it is conceivable that these two enzymes operate by an analogous one-step concerted mechanism. Sequence alignments indeed indicate Glu442 in these enzymes to be equivalent to the proposed active site base (Glu417) in BBE (Supplementary Fig. 7 online). The actual role of these residues, however, will have to be demonstrated by mutagenesis and/or structural studies.

\section{Acknowledgements}

We appreciate the support of staff scientists at the synchrotron beamlines at DESY/EMBLHamburg during diffraction data collection as well as of Hansjörg Weber (Institute of Organic Chemistry, Graz University of Technology) for recording the NMR-spectra. Financial support was provided by the Austrian Science Fund (FWF) through the DK "Molecular enzymology" W901-B05 (to KG and PM).

\section{References}

1. Steffens, P., Nagakura, N. \& Zenk, M.H. Phytochem. 24, 2577-2583 (1985).

2. Kutchan, T.M. \& Dittrich, H. J. Biol. Chem. 270, 24475-24481 (1995).

3. Edmondson, D.E., Binda, C. \& Mattevi, A. Arch. Biochem. Biophys. 464, 269-276 (2007).

4. $\quad$ Scrutton, N.S. Nat. Prod. Rep. 21, 722-730 (2004).

5. $\quad$ Fitzpatrick, P.F. Bioorg. Chem. 32, 125-139 (2004).

6. Winkler, A., Hartner, F., Kutchan, T.M., Glieder, A. \& Macheroux, P. J. Biol. Chem. 281, 21276-21285 (2006).

7. Huang, C.-H. et al. J. Biol. Chem. 280, 38831-38838 (2005).

8. Alexeev, I., Sultana, A., Mantsala, P., Niemi, J. \& Schneider, G. Proc. Natl. Acad. Sci. U. S. A. 104, 6170-6175 (2007). 
9. Heuts, D.P.H.M., Winter, R.T., Damsma, G.E., Janssen, D.B. \& Fraaije, M.W. Biochem. J. 413, 175-183 (2008).

10. Rand, T., Qvist, K.B., Walter, C.P. \& Poulsen, C.H. FEBS J. 273, 2693-2703 (2006).

11. Harayama, T., Tezuka, Y., Taga, T. \& Yoneda, F. J. Chem. Soc., Perkin Trans. 1, 7583 (1987).

12. Iwata, M., Bruice, T.C., Carrell, H.L. \& Glusker, J.P. J. Am. Chem. Soc. 102, 5036-44 (1980).

13. Settembre, E.C. et al. Biochemistry 42, 2971-2981 (2003).

14. Malito, E., Coda, A., Bilyeu, K.D., Fraaije, M.W. \& Mattevi, A. J. Mol. Biol. 341, 1237-1249 (2004).

15. Miller, J.R. \& Edmondson, D.E. Biochemistry 38, 13670-13683 (1999).

16. Bjorklund, J.A. et al. J. Am. Chem. Soc. 117, 1533-1545 (1995).

17. Winkler, A., Kutchan, T.M. \& Macheroux, P. J. Biol. Chem. 282, 24437-24443 (2007).

18. Sirikantaramas, S. et al. J. Biol. Chem. 279, 39767-39774 (2004).

19. Taura, F. et al. FEBS Lett. 581, 2929-2934 (2007). 


\section{Legends for display items}

Figure 1: (a) Overall reaction catalyzed by $\mathrm{BBE}^{1}$. (b) Schematic representation of the protein structure. The N-terminal FAD-binding subdomains are shown in blue and green (including the C-terminal $\alpha$-helical stretch in light green), the central substrate binding domain in magenta. N-linked sugar residues (blue) and the FAD cofactor (orange) are represented as stick models. The amino acids involved in the bi-covalent linkage of FAD are shown in green. (c) Active site environments of the structures from the monoclinic crystals showing polar amino acids as dark green stick models. Alternate conformations were observed for Glu417. The flavin cofactor is shown in orange with its dual mode of attachment to the protein backbone via His104 and Cys166 represented in green. (d) Interactions between the substrate and active site amino acids (green). The substrate is shown in yellow and FAD in orange. Distances are indicated in $\AA$.

Scheme 1: Proposed reaction mechanism for the BBE catalyzed reaction. Reduction of the cofactor is initiated by deprotonation of C3'-OH by Glu417. This increases the nucleophilic behavior of the aromatic ring allowing C-C bond formation in a Friedel-Crafts like alkylation reaction. The formal leaving group, a hydride, is at the same time incorporated into the flavin cofactor. Rearomatization of the intermediate substance leads to $(S)$-scoulerine and might occur spontaneously in solution or could be assisted by another active site base (depicted as B). Regeneration of the cofactor is accomplished by reaction with molecular oxygen and generation of hydrogen peroxide.

Table 1: Summary of the kinetic parameters obtained for active site muteins in comparison to wild-type $\mathrm{BBE}^{\mathrm{a}}$. 
Table 1: Summary of the kinetic parameters obtained for active site muteins in comparison to wild-type $\mathrm{BBE}^{\mathrm{a}}$.

\begin{tabular}{|c|c|c|c|c|}
\hline & Wild-type & Y106F & E417Q & H459A \\
\hline $\boldsymbol{k}_{\text {cat }}\left[\mathrm{s}^{-1}\right]$ & $8.0 \pm 0.2^{\mathrm{b}}$ & $0.7 \pm 0.1$ & $0.054 \pm 0.006$ & $3.1 \pm 0.7$ \\
\hline $\boldsymbol{k}_{\text {red }}\left[\mathrm{s}^{-1}\right]$ & $103 \pm 4^{\mathrm{c}}$ & $6.7 \pm 0.6$ & $0.067 \pm 0.007$ & $88 \pm 4$ \\
\hline $\boldsymbol{k}_{\mathbf{0 x}} \times 10^{5}\left[\mathrm{M}^{-1} \mathrm{~s}^{-1}\right]$ & $0.5 \pm 0.1^{\mathrm{c}}$ & $0.32 \pm 0.03$ & $0.53 \pm 0.02$ & $0.29 \pm 0.03$ \\
\hline
\end{tabular}

${ }^{\text {a }}$ Steady-state turnover rates are shown as mean \pm maximal error of two independent measurements. Reductive and oxidative rates were measured four and five times, respectively, and are stated as mean \pm standard deviation. All kinetic data were measured at $\mathrm{pH} 9$, the previously determined $\mathrm{pH}$ optimum of $\mathrm{BBE}^{1}$.

${ }^{\mathrm{b}}$ data from ref. 6

${ }^{\mathrm{c}}$ data from ref. 17 
a

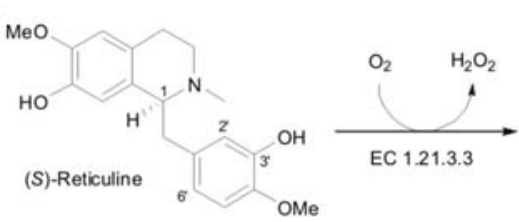

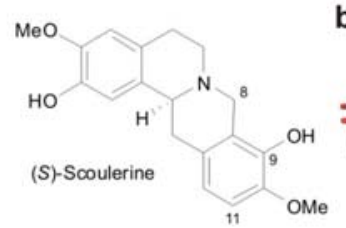

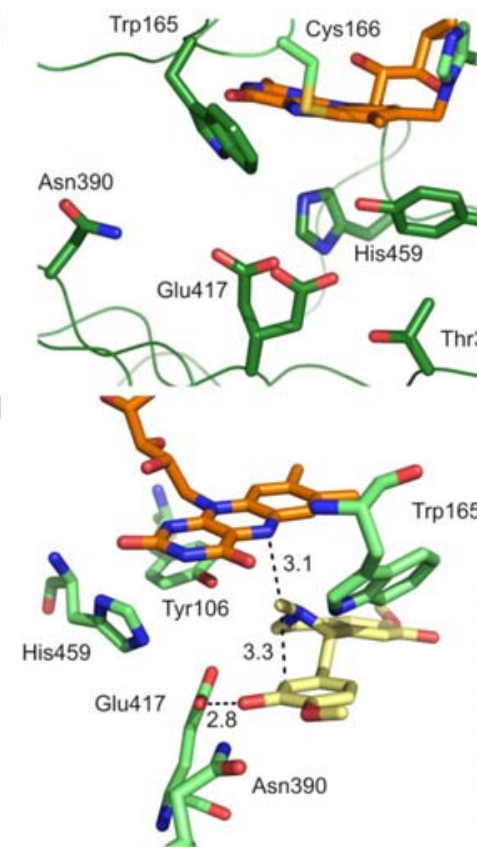

Figure 1: (a) Overall reaction catalyzed by BBE. (b) Schematic representation of the protein structure. The $\mathrm{N}$-terminal FAD-binding subdomains are shown in blue and green (including the $\mathrm{C}$-terminal a-helical stretch in light green), the central substrate binding domain in magenta. $\mathrm{N}$-linked sugar residues (blue) and the FAD cofactor (orange) are represented as stick models. The amino acids involved in the bi-covalent linkage of FAD are shown in green. (c) Active site environments of the structures from the monoclinic crystals showing polar amino acids as dark green stick models. Alternate conformations were observed for Glu417. The flavin cofactor is shown in orange with its dual mode of attachment to the protein backbone via His 104 and Cys 166 represented in green. (d) Interactions between the substrate and active site amino acids (green). The substrate is shown in yellow and FAD in orange. Distances are indicated in $\AA$.

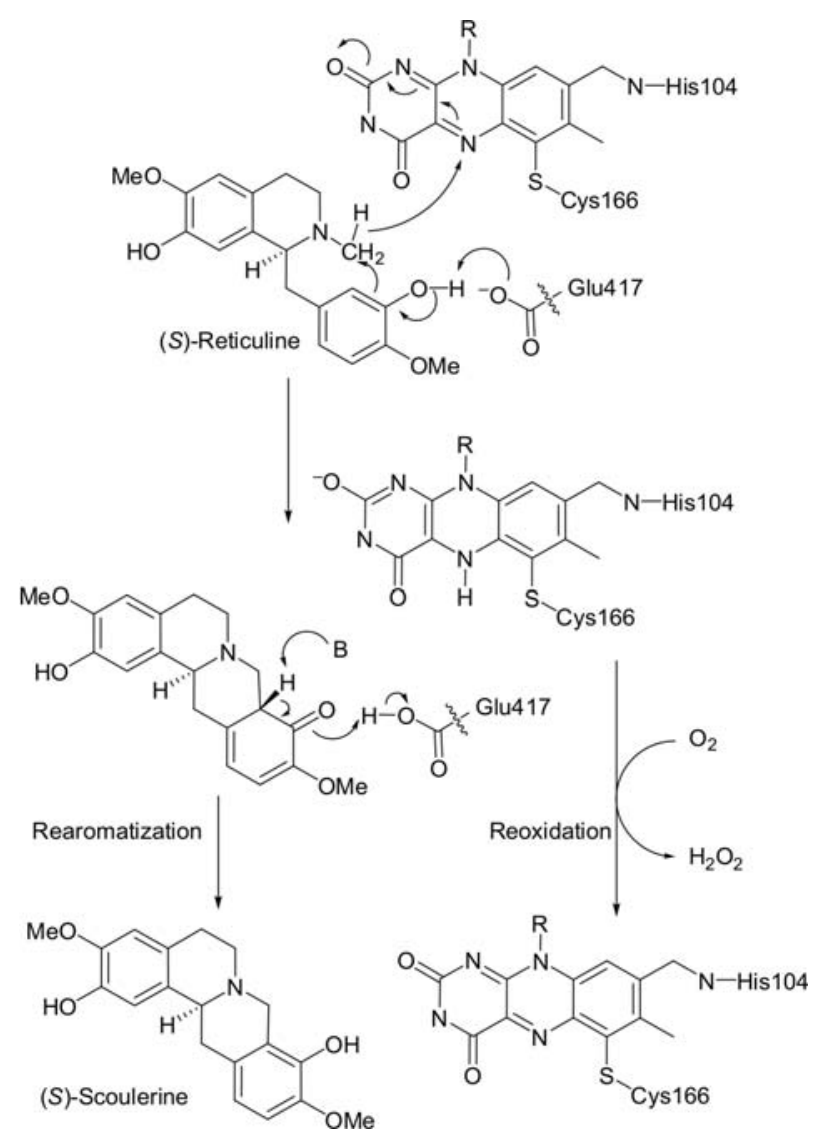

Scheme 1: Proposed reaction mechanism for the BBE catalyzed reaction. Reduction of the cofactor is initiated by deprotonation of $\mathrm{C}^{\prime}-\mathrm{OH}$ by Glu417. This increases the nucleophilic behavior of the aromatic ring allowing $\mathrm{C}-\mathrm{C}$ bond formation in a Friedel-Crafts like alkylation reaction. The formal leaving group, a hydride, is at the same time incorporated into the flavin cofactor. Rearomatization of the intermediate substance leads to $(S)$-scoulerine and might occur spontaneously in solution or could be assisted by another active site base (depicted as B). Regeneration of the cofactor is accomplished by reaction with molecular oxygen and generation of hydrogen peroxide. 